\title{
Workshop Report: Establishing Priority Research Areas to Improve the Physical Health and Well-Being of Autistic Adults and Older People
}

\author{
Georgina Warner, MA, PhD, Jeremy R. Parr, MBChB, MD, and James Cusack, MA, $\mathrm{PhD}^{1}$
}

\begin{abstract}
Many serious medical conditions are more common in autistic adults than the general population. Yet, improving the physical health and well-being of autistic adults has not been a significant research focus to date. In collaboration with Newcastle University (the United Kingdom), Autistica (the United Kingdom's autism research charity) hosted a workshop bringing together autistic people, relatives, clinicians, managers, clinical commissioners, international researchers, and funders to establish priority research areas and catalyze research to improve the physical health and well-being of autistic people. This article provides an overview of the design and methods used at the workshop to maximize collaborative working, and summarizes the 11 priority research concepts that emerged.
\end{abstract}

Keywords: well-being, priority research, autism, autistic adults, physical health

\section{Lay Summary}

Many serious medical conditions are more common in autistic adults than in the general population. Yet, improving the physical health and well-being of autistic adults has not been a significant research focus to date. Autistica (the autism research charity in the United Kingdom [UK]) and clinical researchers from Newcastle University (the UK) identified a need to establish priority research areas and encourage research to improve the physical health and well-being of autistic people. To achieve this aim, both parties cohosted a workshop that brought together autistic people, relatives, clinicians, managers, clinical commissioners, international researchers, and funders from Europe, Australia, and the United States. Thirty-nine people attended the 2day workshop, which was held at a venue with an appropriate environment for autistic people. Eleven research concepts that might be used to improve the physical health and well-being of autistic people were identified:

1. Tailoring of existing measures of pain to better suit autistic people.

2. An international collaborative effort to agree a core set of demographic, health behavior and health outcome indicators most relevant to autistic people that can be compared with general population data.

3. Investigation of health care self-advocacy strategies and potential barriers to effective health care self-advocacy for autistic people.

4. Development of a tool to improve understanding of how factors influence personal well-being for autistic people.

5. A review of evidence on autism-specific health service accommodations and service design to inform what an autism-friendly health service looks like.

6. Adaptation of an online health care "toolkit" developed in the United States to facilitate the primary health care of autistic adults for use in the UK.

7. Development and evaluation of a personalized annual health check program for autistic people.

8. Evaluation of the types of cardiovascular and gut problems most prominent in older autistic adults, contributory factors, and treatment effectiveness.

9. Engagement with autistic people regarding opportunities to use knowledge about genetics and biology to improve health and well-being.

10. Exploration of the research priorities regarding sexual development and health in autism.

11. Investigation of autistic people's use and experiences of residential facilities for older people.

\footnotetext{
${ }^{1}$ Autistica, London, United Kingdom.

${ }^{2}$ Institute of Neuroscience, Newcastle University, Newcastle upon Tyne, United Kingdom.
}

(C) Georgina Warner et al., 2018; Published by Mary Ann Liebert, Inc. This Open Access article is distributed under the terms of the Creative Commons License (http://creativecommons.org/licenses/by/4.0), which permits unrestricted use, distribution, and reproduction in any medium, provided the original work is properly cited. 
The research concepts require further refinement and development. Measuring the impact of the workshop against the primary aim of encouraging research to improve the physical health and well-being of autistic people will take some years. Should one or more projects lead to new knowledge and services that improve the health and well-being of autistic adults and older people, the workshop will have been a success.

\section{Introduction}

\section{The need for physical health and well-being research}

$\mathbf{I}_{\mathrm{tis}}^{\mathrm{N}}$ MPROVING THE PHYSICAL HEALTH and well-being of autistic adults has not been a significant research focus to date. On average, autistic people die $>16$ years earlier than nonautistic people, and for those autistic people with a learning disability, this figure rises to 30 years. ${ }^{1}$ Many serious medical conditions are more common in autistic adults than the general population, including seizure, stroke, gastrointestinal disease, immune conditions, sleep disorders, and diabetes. $^{2}$ Nearly three in four UK autistic people and families think that autistic people receive worse health care than nonautistic people. ${ }^{3}$

The number of research studies dedicated to investigating the physical health of autistic adults remains in the low single figures. ${ }^{4}$ This handful of studies has been conducted solely in the United States and has primarily relied on reviewing the health records of autistic people - a method the researchers acknowledge as having severe limitations. ${ }^{4}$

\section{A community-based participatory research approach}

Patient and public involvement has increasingly become the focus of attention in health research; however, many adopted methods do not facilitate meaningful involvement. ${ }^{5}$ The UK National Institute for Health Research INVOLVE guidelines (www invo.org.uk) describe effective involvement as research "with" or "by" service users, rather than "to," "about," or "for" service users. Essentially, the community should be meaningfully involved in the design of research. Autistica (the UK's autism research charity) is internationally recognized as a leader in this area of involvement, with it being a core value of the organization. ${ }^{6}$ At Newcastle University, autism researchers have also been at the forefront of involving people on the autism spectrum in codesigning and conducting research for many years. ${ }^{7-10}$

In collaboration with Newcastle University, Autistica hosted a workshop bringing together autistic people, relatives, clinicians, managers, clinical commissioners, researchers, and funders to establish priority research areas and catalyze research to improve the physical health and well-being of autistic people. This article aims to provide an overview of the design and methods used at the workshop to maximize collaborative working, to summarize the resulting research concepts, and to encourage research groups with the relevant interest and expertise to pursue the research ideas.

\section{Health care context for workshop research concepts}

The workshop was hosted in the UK and had a number of international delegates (from the United States, Europe, and Australia) - therefore, many of the research concepts were developed in the context of universal health care. With the exception of some prescription and equipment charges, the UK National Health Service (NHS) provides free health care to all UK residents, funded through general taxation. The NHS provides comprehensive health care-including health screening (antenatal screening and routine screenings), treatments for long-term conditions, emergency treatment, and end-of-life care.

\section{Overview of the Workshop}

\section{Aims and ethos}

Autistica collaborative workshops aim to enable autistic people and relatives to come together with multidisciplinary researchers and professionals to cocreate prospective research designs to accelerate previously underinvestigated research areas. The workshops not only put autistic people at the center of research design, they also foster collaboration across disciplines, institutions, and nations. By bringing the leading experts together with people from the autism communities, research can move forward in an impactful, innovative, and efficient way.

The workshop agendas purposefully place a strong focus on establishing future research concepts. For all concerned, it is important to ascertain what is already known in a research field to prevent duplication of effort. However, only a small portion of the workshop agenda is dedicated to reflectionthe primary activity is facilitated group discussions on future possibilities and priorities. The workshops are independently facilitated to maximize the chance that everyone in attendance contributes, and that all have an equal voice. The facilitators do not have subject knowledge, which enhances their ability to objectively take control of the workshop process and enables participants to trust the process as "fair" given that the facilitators have no connection to a research institution or funding organization.

\section{Workshop design}

Thirty-nine people attended the 2-day workshop that was held at a venue with an appropriate environment for autistic people-artificial lighting was avoided where possible, noise was kept to a minimum (e.g., British Sign Language for applause was used in place of clapping), and a quiet room was available for the duration of the workshop. In the first session, after brief introductions (given verbally and in writing, on posters), the group worked together to consider what physical health and well-being research has already been conducted. Brief presentations set out the extent of the problem and stimulated thinking. The group was then encouraged to generate creative research ideas and questions. The ideas and questions were then clustered into common areas of interest. Research areas were subsequently prioritized by the group regarding their potential impact and feasibility. Using a PICO format (Table 1), potential research designs were cocreated in small groups for 
TABle 1. PICO Model

P Patient, Who are the users, patients, or population community? What are their or problem characteristics? What is the condition or disease of interest?

I Intervention What do you want to do with or for the or exposure patient (e.g., treat, diagnose, and observe)?

C Comparison

O Outcome
Is there a control group or comparison (e.g., different treatment options)?

What do you want to achieve through the study? What do you hope to change or measure? each of the prioritized ideas - and the content briefly presented verbally and visually. The expertise of the wider group was drawn upon regarding how the potential research designs could be improved. Finally, with the feedback in mind, the research ideas were further refined (Fig. 1).

\section{Presentations}

The workshop began with a presentation by Cos Michael, an autistic consultant and trainer, who set the context for the event by presenting a number of challenges faced by autistic people that could be adversely affecting their health. Paul Shattuck, Director of the Life Course Outcomes Program at Drexel University, gave an overview of the population-level health indicators research his team has conducted in the

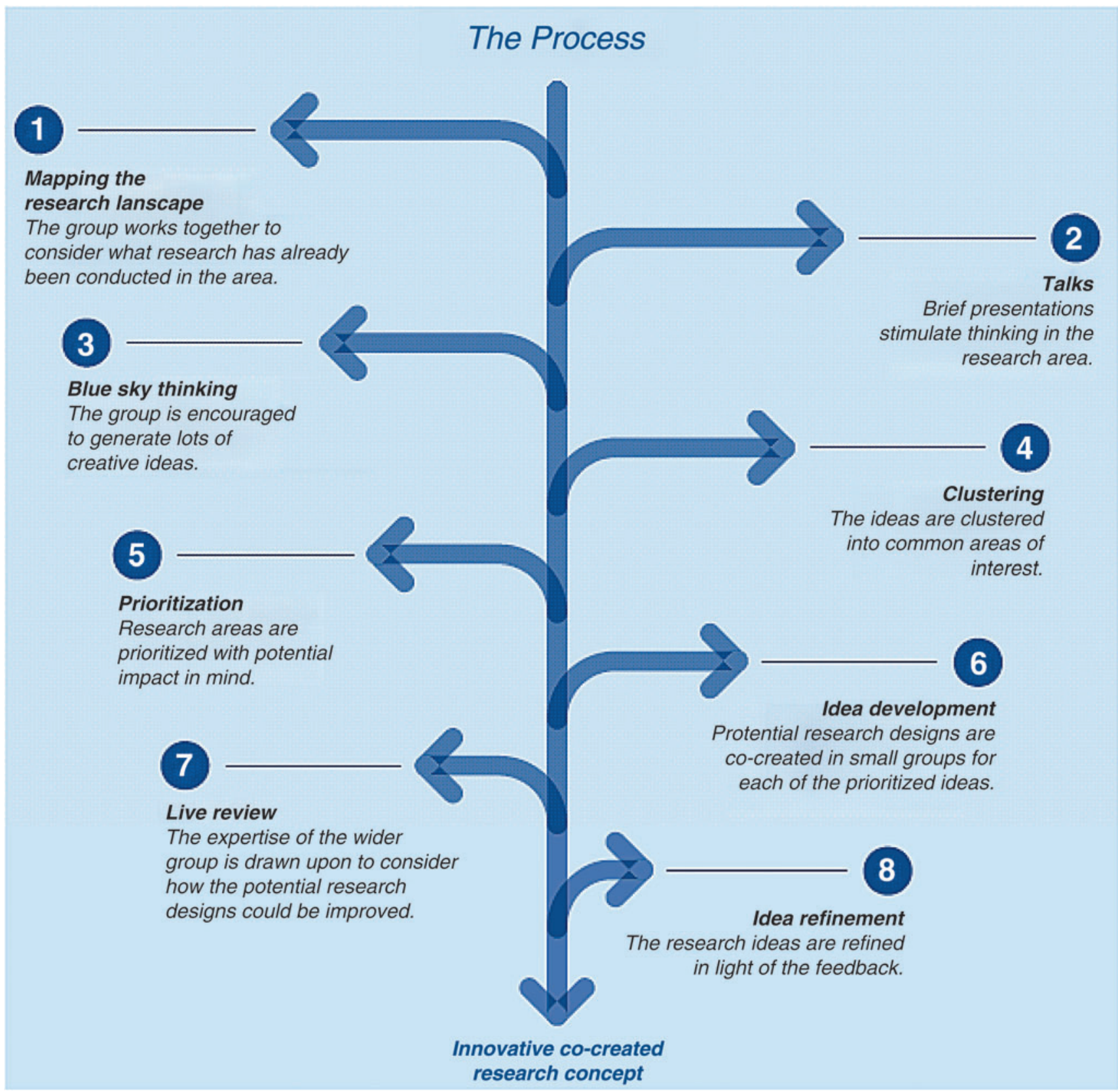

FIG. 1. Autistica collaborative workshop process. 
United States. Christina Nicolaidis, Professor of Social Determinants of Health at Portland State University and Co-Director of the Academic Autism Spectrum Partnership in Research and Education (AASPIRE), spoke of the need to consider community priorities as well as scientific advancement and study feasibility. Jeremy Parr, Autism Spectrum Lifecourse: Adulthood and Ageing Programme Lead at Newcastle University, set out opportunities for "usual health care" to make a difference to the health of autistic people, including new technologies such as personalized medicine (including genetics in health care).

\section{Research Priority Concepts Generated at the Meeting}

The workshop generated 11 research concepts that might be used to improve the physical health and well-being of autistic people. Each of the priority concepts is listed hereunder (in no particular order) - including the rationale for their study, and some initial design elements. A number of other research areas were discussed but not taken forward to the research design stage (Table 2); discussions about research in these areas could take place in the future.

\section{Measurement of pain}

The validity of existing measures of pain for autistic people was raised as an important area to explore. Research into pain in autism has yielded mixed findings, with perceptions of reduced pain sensitivity more recently challenged by perceived differences in pain expression. ${ }^{11}$ Without accurate understanding of pain in autism, assessment of appropriate health interventions and outcomes is challenging.

A study to inform the tailoring of existing measures of pain to better suit autistic people was proposed at the workshop. The study would involve a large scale survey to establish levels of perceived pain in autistic adults compared with the general population. Clinical assessment and investigations could then be used to enable the comparison of selfreport measures with clinical opinion. The research might initially focus on one condition, such as osteoarthritis, but the wider application of the work would be improved measurement of self-reported pain in autism.

\section{Social determinants of health}

Social factors, such as early years experiences, education, employment, income, and environments, and health behaviors including smoking, alcohol consumption, and diet, affect health outcomes in the general population. ${ }^{12}$ We do not know how these factors affect morbidity and mortality rates for

Table 2. Other Areas of Interest Discussed AT THE WORKSHOP

Common co-occurring conditions, e.g., epilepsy

Menopause in autism

Dementia in autism

Sleep management

Immune system function

The role of diagnostic reports in improving access to health care

Social value/cost-effectiveness of improved health and well-being autistic people. A significant barrier to this understanding is the lack of high-quality evidence about the lives of autistic adults and older people.

An important step forward is to agree a core set of socioeconomic indicators most relevant to autistic people that can be compared with general population data across international autism research groups. The project would scope existing autism research efforts to utilize population level indicators/ measures. Subsequently, an international autism research collaboration would establish a core set of demographic, health behavior, and health outcomes - then there would be an assessment of the reliability and validity of the identified indicators/measures for autistic people. The project would provide high-quality data regarding the similarities and differences in terms of social determinants of health in autistic adults and older people, compared with the general population. This would lead to specific data about autistic people and inform priority areas for intervention. The information and subsequent research would positively impact service provision and policy and may lead to improvements in the health of autistic people.

\section{Health care self-advocacy}

There are many perceived benefits of health care selfadvocacy, including a more patient-centered approach to care; improved patient-provider relationship, self-care, and health outcomes; more efficient health care delivery; and reduced health care costs. ${ }^{13}$ However, health care selfadvocacy strategies and potential barriers to effective selfadvocacy for autistic people have not been explored yet.

Workshop attendees advised conducting a review of scientific literature alongside a modified Delphi consultation-a group communication method that enables prioritization-to establish existing "best practice" to improve patient selfadvocacy with regard to accessing health care. A qualitative approach would then be used to identify strategies that have worked or might do so for the majority of autistic people. An exemplar approach for health care self-advocacy for autistic people, such as a "roadmap"- a visual representation that organizes and presents important information related to future plans-would be developed and trialed for feasibility and acceptability. The project could improve autistic people's ability to prospectively obtain quality health care; this might lead to generalization, and a wider application of improved self-advocacy in other areas of autistic people's lives.

\section{Well-being in autism}

Well-being may have a protective role in health maintenance, with sense of purpose and meaning in life being associated with increased survival in the general population. ${ }^{14}$ However, factors influencing well-being may differ for autistic people. Improved understanding of how factors influence personal well-being for autistic people was proposed at the workshop. The project would involve a review of scientific literature regarding autism and well-being alongside secondary analysis of existing data sets to identify factors associated with well-being in autism. Consultation with autistic adults and service providers would then take place, with the view to developing a personalized "well-being tool" that records individual views and experiences against factors associated with well-being in autism. A feasibility and acceptability trial of the well-being tool would be conducted, 
including an investigation of the economic value of the tool. The tool could improve understanding of autistic people's personal well-being baselines and the ability to identify ways to improve personal well-being. It could enable, and provide a standardized process for, health care providers to develop more focused personal support plans.

\section{Autism-friendly services}

Before an effective program of practitioner education regarding health care provision for autistic people can be developed, it was argued that we must first understand what an autism-friendly health service looks like. A scoping review was proposed to collate evidence on autism-specific health service accommodations and design. The review would include learning from wider groups, such as learning disability and dementia. A modified Delphi consultation-a group communication method that enables prioritization-would be used to gather the views of autistic adults, families, service providers, and third sector organizations on health care provision for autistic people. This project would develop a vision for best practice in how to provide health care to autistic people; identify gaps in practitioner knowledge, skills, and values; and identify strategies to change service models to enable an autism-friendly health service.

\section{Toolkits to improve health and well-being}

In the United States, a community-based participatory research approach has been used to develop and evaluate an online health care "toolkit" to facilitate the primary health care of autistic adults. ${ }^{15}$ Initial testing of the toolkit demonstrated it was easy for autistic adults to use-it helped them to prepare for health visits, decreased the overall number of barriers to their health care, and improved communication with their health care providers. ${ }^{15}$ A UK-U.S. collaboration to adapt the toolkit content for use in the UK context was discussed at the workshop. The project would involve a feasibility trial of the integration of the toolkit into NHS clinical services, including investigation of the mechanism of action achieved by the toolkit and exploration of how to enable ongoing quality improvement of the resource. As demonstrated by the work in the United States, the toolkit could improve autistic people's ability to obtain quality health care in the UK, as well as improve experiences of health care.

\section{Annual health checks}

Annual health checks for adults with learning disability have been incentivized by NHS England since 2009. The health checks were intended to identify undetected health problems and improve prescribing and coordination with secondary care. Systematic reviews have confirmed they are effective in identifying new health problems, and improving uptake of preventive interventions and indicators of process of care. ${ }^{16}$

The development and evaluation of a similar annual health check program for autistic people were discussed at the workshop. The project would involve a review of scientific literature to collate evidence on the utilization of health checks, a qualitative approach to identify the opinions of autistic adults and health care professionals, and a national survey to gather a wider perspective. A multidisciplinary team would be assembled to create the health check-they would work in partnership with NHS organizations and structures. A feasibility and acceptability trial of the health check would be conducted, including investigation of training requirements and preferred location for use (e.g., general practitioner [GP] practice versus an autism-specific service). Autism annual health checks could increase access to health care, improve the health of autistic people-reducing morbidity and early mortality-improve health care experiences, and lower the cost of health care through preventative action.

\section{Priority physical health areas}

Many chronic medical conditions that are associated with significant morbidity and early mortality rates are more common in autistic adults than in the general population. ${ }^{2}$ Of these conditions, cardiovascular problems and their causes, and gut/bowel problems were identified as priorities.

A research project evaluating the types of cardiovascular and gut problems that are most prominent in older autistic adults, contributory factors, and treatment effectiveness was proposed. A review of scientific literature would be conducted to understand what is known about the prevalence and treatment effectiveness of cardiovascular and gut problems in (1) autistic adults, (2) other neurodevelopmental conditions, and (3) the general population. An international prevalence study of specific cardiovascular and gut conditions in autistic adults would be conducted, alongside consultation with autistic people and health care specialists to understand potential autismspecific contributory factors. The project aims to improve understanding and detection of cardiovascular and gut problems-priority physical health concerns for autistic adults.

\section{Personalized health care (making genetics and biology work for autistic people)}

Personalized health care is considered the key opportunity to improve health during the 21 st century. ${ }^{17}$ There are opportunities for translational biology and genetics to facilitate a personalized health care approach. ${ }^{18}$ However, it was acknowledged at the workshop that many autistic people have reservations about autism genetic research, as one view is that research exists to inform a "cure" for autism, or to reduce the number of autistic people.

Therefore, an engagement project around this topic was proposed. A mixed method approach would be taken to consult with autistic people regarding opportunities to use knowledge about biology to improve health and well-beingthis could include workshops, surveys, and qualitative interviews. The discussions would be placed in the context of how genetics, as well as drug development and personalized treatment, is improving the lives of people with other conditions. The project's aim would be to publish a strategy for the development of personalized health care and stratified medicine, mutually agreed by autistic people and relatives, clinicians, and researchers. This platform would then be built upon by new research projects aiming to improve the health of autistic people.

\section{Sexual development}

There are several health issues relating to adolescent sexual orientation. Sexual minority youth report higher rates of 
mental health issues (e.g., depression, self-harm, and suicidal ideation) and higher rates of sexually transmitted diseases than their heterosexual peers. ${ }^{19}$ Compared with the general population, there is emerging evidence that more autistic people report sexual attraction to both same- and oppositesex partners. ${ }^{20}$

A project exploring the research priorities regarding sexual development in autism was proposed at the workshop. A review of scientific literature, qualitative research methods, and online content analysis would be used to understand what is known about sexual development in autism and how this may relate to health. The project would improve understanding and awareness of issues related to sexual development among autistic people and health professionals and improve access to relevant services.

\section{Residential care in older age}

Making the transition from one's home to residential care can be stressful, evoking feelings of uncertainty, a lack of control, and a loss of identity. ${ }^{21}$ The residential environment itself can affect well-being. ${ }^{22}$ However, we know little about autistic people's experiences of transferring to and living in residential care.

A project investigating the current use of residential facilities and autistic people's experiences was proposed. Archival and survey data would be used to calculate general descriptive statistics regarding how many autistic people utilize residential care facilities for older adults. The type of facilities used, and peoples age and health status would be investigated (in comparison with same age people in the general population). A qualitative approach would be used to consider older autistic adults' experiences of residential care and potential strategies to improve the care experience and support autonomy where possible. The project could inform future interventions, resources, and policies to improve the health and well-being of autistic adults who may require residential care in older age (e.g., through improved facilities and reasonable adjustments to care).

\section{Discussion}

We have described how a collaborative workshop that acknowledges and utilizes the expertise and experience of a range of participants can identify research priorities and initial research designs. The meeting led to 11 priority concepts, which can be used by researchers to design future studies-the research concepts are not the intellectual property of delegates, but can be pursued by research groups with the relevant interest and expertise.

\section{Content of output}

It is acknowledged that collaborative workshops can catalyze new research in under-researched areas but are only the beginning of the process. The research designs generated at the workshop represent tangible research ideas that can be taken forward, but require further refinement and will be at varying stages of development. It will be important to establish the detail of what is already known regarding each of the research concepts. Many of the proposed projects include initial reviews and/or consultations-it is likely these will inform the refinement of the research concepts, and so a staged approach should be taken in driving these ideas forward.

There was considerable crossover among the workshop outputs. For instance, resources such as the online health care toolkit and the personal well-being tool could influence and inform research into self-advocacy and/or annual health checks. Health care context must also be considered-as many of the research concepts were developed in the U.K. context of universal health care.

\section{Impact}

Measuring the impact of the workshop against the primary aim of catalyzing research to improve the physical health and well-being of autistic people will take some years. It can take many years for research evidence to reach clinical practice. The creation of knowledge does not, of itself, lead to widespread implementation and positive impacts on health-the knowledge must be translated into changes in practice and policy for the benefits to flow to patients. Eleven tangible research concepts have been generated-should one or more projects lead to new knowledge and services that improve the health of autistic adults and older people, the workshop will have been a success. Autistica is committed to taking the research concepts forward and funds have already been secured to develop and trial a personalized health checks program for autistic adults. There are several other metrics of success that can be applied to the workshop, including greater use of methods that facilitate meaningful involvement of autistic people in research design and increased collaboration across international autism research groups.

\section{Acknowledgments}

The collaborative workshop was funded by Autistica, the national autism research charity in the UK, hosted in collaboration with members of the Autism Spectrum Lifecourse Adulthood and Ageing Research Programme and other colleagues from Newcastle University. The workshop was facilitated by The Collective Facilitation Ltd.

\section{Authorship Confirmation Statement}

Dr. Georgina Warner, Research Manager at Autistica, led on writing the article with contributions from Dr. Jeremy Parr, Autism Spectrum Lifecourse: Adulthood and Ageing Programme Lead at Newcastle University, and Dr. James Cusack, Director of Science at Autistica. All coauthors reviewed and approved the article before submission. The article has been submitted solely to Autism in Adulthood and is not published, in press, or submitted elsewhere.

\section{Author Disclosure Statement}

The collaborative workshop was funded by Autistica (Dr. Georgina Warner and Dr. James Cusack) and hosted in collaboration with members of the Autism Spectrum Lifecourse Adulthood and Ageing Research Programme (Dr. Jeremy Parr). There are no other conflicts of interest to declare. 


\section{References}

1. Hirvikoski T, Mittendorfer-Rutz E, Boman M, Larsson H, Lichtenstein P, Bölte S. Premature mortality in autism spectrum disorder. Br J Psychiatry. 2016;208(3):232-238.

2. Croen LA, Zerbo O, Qian Y, et al. The health status of adults on the autism spectrum. Autism. 2015;19(7):814-823.

3. The Westminster Commission on Autism. A Spectrum of Obstacles: An Inquiry into Access to Healthcare for Autistic People. United Kingdom: National Children's Group; 2016.

4. Cashin A, Buckley T, Trollor JN, Lennox N. A scoping review of what is known of the physical health of adults with autism spectrum disorder. J Intellect Disabil. 2016; 22(1):96-108.

5. Tierney E, McEvoy R, O'Reilly-de Brún M, et al. A critical analysis of the implementation of service user involvement in primary care research and health service development using normalization process theory. Health Expect. 2016; 19(3):501-515.

6. Autistica. Involvement promise. 2017. www.autistica.org. uk/downloads/files/Autistica-Involvement-Promise.PDF; Last accessed February 13, 2018.

7. McConachie H, Barry R, Spencer A, Parker L, Le Couteur A, Colver A. Daslne: the challenge of developing a regional database for autism spectrum disorder. Arch Dis Child. 2009;94(1):38-41.

8. McConachie H, Mason D, Parr JR, Garland, D, Wilson C, Rodgers J. Enhancing the validity of a quality of life measure for autistic people. J Autism Dev Disord. 2017 [Epub ahead of print]; DOI: 10.1007/s10803-017-3402-z.

9. Parr JR. How can we learn more about the lives of adults on the autism spectrum, and their relatives? In Wright SD, ed. Autism Spectrum Disorder in Mid and Later Life. Philadelphia, Pennsylvania: Jessica Kingsley Publishing; 2016.

10. Warnell F, George B, McConachie H, Johnson M, Hardy R, Parr JR. Designing and recruiting to UK autism spectrum disorder research databases: do they include representative children with valid ASD diagnoses? BMJ Open. 2015;5(9): e008625.

11. Allely CS. Pain sensitivity and observer perception of pain in individuals with autistic spectrum disorder. ScientificWorldJournal. 2013;2013:916178.

12. Marmot M, Allen JJ. Social determinants of health equity. Am J Public Health. 2014;S517-S519.
13. Hagan TL, Rosenzweig MQ, Zorn KK, van Londen GJ, Donovan HS. Perspectives on self-advocacy: Comparing perceived uses, benefits, and drawbacks among survivors and providers. Oncol Nurs Forum. 2017;44(1):52.

14. Steptoe A, Deaton A, Stone AA. Subjective wellbeing, health, and ageing. Lancet. 2015;385(9968):640-648.

15. Nicolaidis C, Raymaker D, McDonald K, et al. The development and evaluation of an online healthcare toolkit for autistic adults and their primary care providers. $J$ Gen Intern Med. 2016;31(10):1180-1189.

16. Robertson J, Hatton C, Emerson E, Baines S. The impact of health checks for people with intellectual disabilities: an updated systematic review of evidence. Res Dev Disabil. 2014;35(10):2450-2462.

17. Grech G, Grossman I, eds. Preventive and Predictive Genetics: Towards Personalised Medicine (Vol. 9). Switzerland: Springer; 2015.

18. Vorstman JA, Parr JR, Moreno-De-Luca D, Anney RJ, Nurnberger Jr. JI, Hallmayer JF. Autism genetics: opportunities and challenges for clinical translation. Nat Rev Genet. 2017;18(6):362-376.

19. Saewyc EM. Research on adolescent sexual orientation: Development, health disparities, stigma, and resilience. J Res Adolesc. 2011;21(1):256-272.

20. Dewinter J, De Graaf H, Begeer S. Sexual orientation, gender identity, and romantic relationships in adolescents and adults with autism spectrum disorder. J Autism Dev Disord. 2017;47(9):2927-2934.

21. Lee VS, Simpson J, Froggatt K. A narrative exploration of older people's transitions into residential care. Aging Ment Health. 2013;17(1):48-56.

22. Nordin S, McKee K, Wallinder M, Koch L, Wijk H, Elf M. The physical environment, activity and interaction in residential care facilities for older people: a comparative case study. Scand J Caring Sci. 2016;31(4):727-738.

Address correspondence to: Georgina Warner, MA, PhD

Autistica

St. Saviour's House 39-41 Union Street London SE1 1SD United Kingdom

E-mail: georgina.warner@autistica.org.uk 